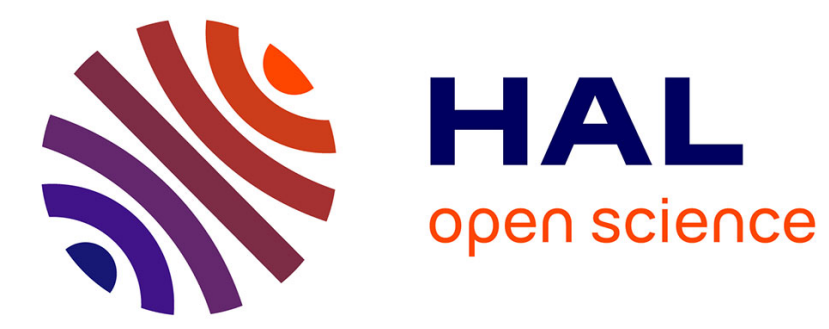

\title{
Satisficing versus optimality: criteria for sustainability
}

\author{
Michel de Lara, Vincent Martinet, Luc Doyen
}

\section{To cite this version:}

Michel de Lara, Vincent Martinet, Luc Doyen. Satisficing versus optimality: criteria for sustainability. Bulletin of Mathematical Biology, 2015, 77 (2), pp.281-297. 10.1007/s11538-014-9944-8 . hal-01123050

\section{HAL Id: hal-01123050 \\ https://hal.science/hal-01123050}

Submitted on 5 Jul 2019

HAL is a multi-disciplinary open access archive for the deposit and dissemination of scientific research documents, whether they are published or not. The documents may come from teaching and research institutions in France or abroad, or from public or private research centers.
L'archive ouverte pluridisciplinaire HAL, est destinée au dépôt et à la diffusion de documents scientifiques de niveau recherche, publiés ou non, émanant des établissements d'enseignement et de recherche français ou étrangers, des laboratoires publics ou privés. 


\title{
Satisficing versus Optimality: Criteria for Sustainability
}

\author{
Michel De LARA, Vincent MARTINET ${ }^{\dagger}$ and Luc Doyen ${ }^{\ddagger}$
}

\begin{abstract}
Economic analysis addresses risk and long-term issues with discounted expected utility, focusing on optimality. Viability theory is rooted on satisfying sustainability constraints over time, focusing on feasibility. We build a bridge between these two approaches by establishing that viability is equivalent to an array of degenerate intertemporal optimization problems. First, we focus our attention on the deterministic case. We highlight the connections between the viability kernel and the minimum time of crisis. Carrying on, we lay out stochastic viability, turning the spotlight onto the notions of viable scenario and maximal viability probability. Our conceptual results bring the viability approach closer to the economic approach, especially in the stochastic case and regarding efficiency. We discuss the possible use of viability as a theoretical framework for biodiversity conservation, ecosystem management and climate change issues.
\end{abstract}

Keywords: sustainability; uncertainty; optimality; viability.

*Université Paris-Est, CERMICS (ENPC), 6-8 Avenue Blaise Pascal, Cité Descartes, F-77455 Marne-la-Vallée. E-mail: delara@cermics.enpc.fr

${ }^{\dagger}$ INRA, UMR210 Economie Publique, F-78850 Thiverval-Grignon, France. E-mail: vincent.martinet@grignon.inra.fr

${ }^{\ddagger}$ CNRS, GREThA, University of Bordeaux 4, Avenue Léon Duguit 33608 Pessac cedex France. Email: luc.doyen@u-bordeaux4.fr 


\section{Introduction}

Dealing with environmental issues - such as biodiversity conservation, ecosystem based fisheries management or climate change - requires us to account for conflicting objectives, dynamics, long-term, irreversibility and uncertainty. The issue of decision-making under uncertainty and risk has been widely addressed in the economic literature, back to the fundament of expected utility theory axiomatized by von Neuman and Morgenstern [1947]. However, the expected utility framework was soon criticized on empirical grounds (e.g., the Allais [1953] and Ellsberg [1961] paradoxes), and a huge literature has developed to propose alternatives (e.g., [Kahneman and Tversky, 1979, Tversky and Kahneman, 1992], to name a few).

Dynamic and long-term issues have also received a particular focus in the economic literature, especially in growth theory [Koopmans, 1965]. Regarding environmental issues, the discounted utility approach is criticized in the sustainability debate: this widespread neo-classical criterion may lead to unsustainable economic trajectories in models of optimal growth with environment [Heal, 1998], mainly because of discounting. The discounted utility criterion neglects long-run utility, and is qualified as a "dictatorship of the present" by Chichilnisky [1996]. The sustainability debate has thus been marked by the introduction and analysis of other criteria [Heal, 1998], including maximin [Solow, 1974].

Howarth [1995] emphasizes that, under uncertainty, a deontological approach should be used to address the sustainability issue, and sustainability conditions should be imposed as prior constraints on the maximization of a social welfare function. Using physical quantities to deal with the sustainability issue, due to its long-term perspective, is an alternative approach to expected utility [Mäler, 2002]. This approach is the one favored to deal with the climate change issue in practice, as illustrated by the Kyoto protocol which defines physical targets in quantitative terms $\left(\mathrm{CO}_{2}\right.$ emissions). Hence many studies consider explicit constraints on GHG (greenhouse gases) emissions or concentrations instead of damage functions as, for instance, in Chakravorty et al. [2008]. In biodiversity conservation, physical constraints are usually prefered to utility evaluations. As an example, the International Council for the Exploration of the Sea (ICES) precautionary approach [ICES, 2004] aims at conserving fish stocks and fisheries on the grounds of several indicators including spawning stock biomass and fishing mortality. In this context, reference points not to be exceeded for these bioeconomic indicators 
stand for management objectives.

When sustainability objectives are defined using indicators (quantitative measurements having an economic or physical meaning) and thresholds (limits not to be exceeded), the problem of the regulator in coping with all the objectives simultaneously is to avoid crisis situations. Hence, sustainability appears closer to a "satisficing" problem than to an optimizing problem (see the discussion by Krawczyk and Kim [2009]). " The concept of "stewardship", mentioned in the Stern Review, also stresses similar issues:

The notion of "stewardship" can be seen as a special form of sustainability. It points to particular aspects of the world, which should themselves be passed on in a state at least as good as that inherited from the previous generation. [Stern, 2006]

If, from an intergenerational equity perspective, the constraints defined by these indicators and thresholds have to be satisfied over time, such sustainability problems can be studied in the mathematical framework of viability theory [Béné et al., 2001, Martinet and Doyen, 2007, Baumgärtner and Quaas, 2009, Péreau et al., 2012, Cissé et al., 2013, Hardy et al., 2013]. A major mathematical instrument of the viability analysis is the so-called viability kernel [Aubin, 1991]. It is composed of all initial states, if they exist, from which economic development paths respecting the constraints can start under appropriate sequences of decisions. Focusing on such a geometrical tool is not traditional in economic analysis. We claim that the mathematical framework of viability theory, with its concepts and methods, is well suited to account for the major ingredients related to "stewardship", "satisficing", or, more broadly, of "sustainability". Our ambition is not to scrutinize the possible acceptances and differences between "stewardship", "satisficing", "sustainability", etc. We just propose one mathematical formalism that can capture the essence of these concepts.

Placed in the discussion on risk, viability can be interpreted in a stochastic framework as the requirement to keep indicators above given thresholds with a high probability [Morris and Doak, 2003, De Lara and Doyen, 2008, Baumgärtner and Quaas, 2009, Doyen and De Lara, 2010, Doyen et al., 2012, Gourguet et al., 2013, Mouysset et al., 2014]. This interpretation revolves

\footnotetext{
${ }^{1}$ The word satisficing inspired by Simon [1957] and bounded rationality does not here refer to a situation of limited information in the decision process but as an alternative to optimality.
} 
around the psychological process of "aspiration assessment": Lopes claims that "sensible people often base their choices on the probability of coming out ahead" [Lopes, 1996], that is, to achieve at least a certain level with high enough probability. When stakes are high, as life and death issues illustrated in Dubbins and Savage [1965], this is a fairly "reasonable" approach. We will discuss those points in the conclusion.

In this paper, we examine how the viability approach, and especially stochastic viability, addresses conflicting objectives, uncertainty, dynamic processes and long-term issues. Our contribution is twofold. First, from a theoretical point of view, we provide a criterion-like description of the viability approach, which allows us to stress its links with the usual economic approach of (expected) discounted utility. In fact, the equivalence is not with one but with several degenerate optimization problems: we provide a description of time additive and time multiplicative criteria, and interpret each kind of formulation. Second, we argue that stochastic viability is a relevant approach to deal with dynamic problems under uncertainty, and thus to cope with long-run environmental issues such as climate change, biodiversity conservation or natural resources management.

The paper is organized as follows. In Sect. 2, we describe both the usual discounted utility and the viability approach in the deterministic setting. We emphasize their conceptual differences, and the implications of these differences for natural resource management issues. In Sect. 3, we depict both approaches in a stochastic framework. We highlight that, when time and risk interact, the differences between approaches reduce and are sources of complementarity, at least to address sustainability issues. We conclude with a discussion in Sect. 4.

\section{Deterministic intertemporal problem}

As stated in the introduction, natural resources management and environmental issues are dynamic in nature. To address these issues, we use a dynamic modeling framework from control theory. We consider management of dynamic systems in discrete time; we indeed want to avoid technical difficulties related to continuous time, and concentrate on conceptual issues. 


\subsection{Dynamic bio-economic model}

We consider the following control dynamical system in discrete-time,

$$
x(t+1)=G_{t}(x(t), c(t)), \quad t=t_{0}, \ldots, T-1, \quad x\left(t_{0}\right)=x_{0},
$$

where the time index $t$ is discrete $\left(t \in \mathbb{N}\right.$ is an integer), with $t_{0}$ the initial time and $T$ the horizon, which may be finite $(T<+\infty)$ or infinite $(T=+\infty)$. The state $x(t)$ is a vector belonging to $\mathbb{X}=\mathbb{R}^{n}$; usually, the components of the state vector $x(t)$ represent stocks; such stocks can include renewable resources (typically abundance, biomass of species), man-made reproducible capital, or pollution stocks; the vector $x_{0} \in \mathbb{X}$ is the initial state for the initial time $t_{0}$. The control $c(t) \in \mathbb{C}=\mathbb{R}^{p}$ represents decisions, such as extraction or harvesting effort, investment, consumption, emissions. The mappings $G_{t}: \mathbb{X} \times \mathbb{C} \rightarrow \mathbb{X}$, for $t=t_{0}, \ldots, T-1$, stand for the dynamics representing the evolution of the state through time. Examples encompass population dynamics, ecosystem dynamics, economic models with capital and labor, or pollution accumulation-absorption models. We now proceed by laying out a model in biodiversity management.

\section{A two-species fisheries model}

We consider a fisheries dynamical model with two species, each targeted by a specific fleet. Each species is described by its biomass: the two-dimensional state vector $\left(x_{1}, x_{2}\right)$ represents the biomasses of both species. The twodimensional control vector $\left(c_{1}, c_{2}\right)$ comprises the harvesting effort for each species, respectively, each lying in $[0,1]$. The discrete-time control dynamical system we consider is given by

$$
\left\{\begin{array}{l}
x_{1}(t+1)=x_{1}(t) \mathcal{R}_{1}\left(x_{1}(t), x_{2}(t)\right)\left(1-c_{1}(t)\right) \\
x_{2}(t+1)=x_{2}(t) \mathcal{R}_{2}\left(x_{1}(t), x_{2}(t)\right)\left(1-c_{2}(t)\right)
\end{array}\right.
$$

The two functions $\mathcal{R}_{1}: \mathbb{R}^{2} \rightarrow \mathbb{R}$ and $\mathcal{R}_{2}: \mathbb{R}^{2} \rightarrow \mathbb{R}$ represent biological growth factors.

As an example of application, in De Lara et al. [2012], the Peruvian anchovy-hake system is modeled as a prey-predator system, where the anchovy growth rate is decreasing in the hake population. We describe this interaction by the following discrete-time Lotka-Volterra system 


$$
\begin{aligned}
& x_{1}(t+1)=x_{1}(t) \overbrace{\left(\begin{array}{c}
\left.R-\frac{R}{\kappa} x_{1}(t)-\alpha x_{2}(t)\right) \\
x_{2}(t+1)
\end{array}=x_{2}(t)\right.}^{\mathcal{R}_{1}\left(x_{1}(t), x_{2}(t)\right)}\left(1-c_{1}(t)\right), \\
& \underbrace{\left(L+\beta x_{1}(t)\right)}_{\mathcal{R}_{2}\left(x_{1}(t), x_{2}(t)\right)}\left(1-c_{2}(t)\right)
\end{aligned}
$$

where $R>1,0<L<1, \alpha>0, \beta>0$ and $\kappa=\frac{R}{R-1} K$, with $K>0$ the carrying capacity for the prey. The variable $x_{1}$ stands for anchovy biomass and $x_{2}$ for hake biomass. The purpose of this compact model is not to provide biological "knowledge" on the Peruvian upwelling ecosystem, but rather to capture the essential features of the system in what concerns decision making.

\subsection{Outputs/indicators}

Main outputs of system (1) are given by so-called indicators $\mathcal{I}_{t}^{k}(x(t), c(t))$, $k=1, \ldots, K$. An indicator $\mathcal{I}_{t}^{k}: \mathbb{X} \times \mathbb{C} \rightarrow \mathbb{R}$ is a state and control function having economic or environmental meaning. ${ }^{2}$ From a sustainable development point of view, the indicators are instantaneous measurement of quantities that characterize some aspects of sustainability (biodiversity, catches, rents, $\mathrm{CO}_{2}$ emissions, consumption, etc.). Note that an indicator can be reduced to the simplest form, being only one of the stocks or one of the decisions (consumption for instance).

In what follows, when we turn the spotlight upon welfare economics formulations, we will consider that the indicators are directly the components of a utility function $U$, forging the value $U\left(\mathcal{I}_{t}^{1}(x(t), c(t)), \ldots, \mathcal{I}_{t}^{K}(x(t), c(t))\right)$ at time $t$. The utility function is thus implicitly relying on time, state and control. For example, in fisheries management, the utility can be derived both from fish stocks (as biodiversity or ecosystem services indicators) and catches, that are important economic indicators. In a climate change context, the utility can be derived both from consumption and from a climate indicator.

\footnotetext{
${ }^{2}$ When the horizon $T<+\infty$, the indicator $\mathcal{I}_{T}^{k}$ only maps the state space $\mathbb{X}$ towards $\mathbb{R}$ because there is no decision at time $T$. However, for the sake of homogeneity of notations, we will write $\mathcal{I}_{T}^{k}(x(T), c(T))$ for $\mathcal{I}_{T}^{k}(x(T))$.
} 


\subsection{Maximal intertemporal discounted utility}

In an intertemporal framework, one purpose of economic analysis is to identify optimal intertemporal decisions paths to be applied from a given initial state.

The usual criterion in economics is the discounted utility, that is, the discounted sum of present and future utilities

$$
\max _{c(\cdot)} \sum_{t=t_{0}}^{T} \frac{1}{(1+\delta)^{t}} U\left(\mathcal{I}_{t}^{1}(x(t), c(t)), \ldots, \mathcal{I}_{t}^{K}(x(t), c(t))\right),
$$

where $\delta>0$ is the discount rate, and $c(\cdot)$ denotes a control path $c(\cdot)=$ $\left(c\left(t_{0}\right), \ldots, c(T-1)\right)$. For instance, in the two-species fisheries model introduced in $\S 2.1$, we can maximize the discounted value of the catches.

A program such as (4) defines an optimal growth path in the terminology of neo-classical economics [Koopmans, 1965]. In the long run, it can lead to unsustainable situations, in the sense of a utility decreasing toward zero, in particular in models with exhaustible natural resources [Dasgupta and Heal, 1974] or pollution.

\subsection{The viability approach}

Following the discussion in Sect. 1, suppose now that the decision maker's goal is not to maximize the discounted utility but to maintain given indicators above viability thresholds: ${ }^{3}$

$$
\mathcal{I}_{t}^{k}(x(t), c(t)) \geq \theta_{t}^{k}, \quad \forall k=1, \ldots, K, \quad \forall t=t_{0}, \ldots, T .
$$

Recall that $\mathcal{I}_{t}^{k}: \mathbb{X} \times \mathbb{C} \rightarrow \mathbb{R}$ is an indicator, namely a state and control function having economic or biological meaning; the real number $\theta_{t}^{k}$ is a threshold. ${ }^{4}$ Using these indicators and associated thresholds acting as constraints, a viable development path is an economic path that meets the constraints (5), that is, that meets the constraints $\mathcal{I}_{t}^{k}(x(t), c(t)) \geq \theta_{t}^{k}$, for all $k=1, \ldots, K$ and at all times $t=t_{0}, \ldots, T$.

\footnotetext{
${ }^{3}$ Without loss of generality: a "bad" indicator, such as pollution, can be represented by its negative value, so that the direction of the inequality holds.

${ }^{4}$ Since the indicator $\mathcal{I}_{t}^{k}$ and the threshold $\theta_{t}^{k}$ are allowed to explicitly depend upon time $t$, we can cover the case of absence of constraints (take $\mathcal{I}_{t}^{k}$ having constant value greater than $\theta_{t}^{k}$ ), or of final target constraint (take $\mathcal{I}_{t}^{k}(x, c)=0$ and $\theta_{t}^{k}=0$ for all $t=t_{0}, \ldots, T-1$, but not for $\mathcal{I}_{T}^{k}(x, c)$ and $\left.\theta_{T}^{k}\right)$.
} 


\section{Viability kernel}

The viability analysis describes the conditions on states (ecological and economic endowments) and controls (economic decisions) for the resulting path to be viable, that is, to respect all the constraints at all times, given the dynamics of the system. The main mathematical instrument of the viability analysis is the so-called viability kernel [Aubin, 1991]. It is composed of all initial states from which viable trajectories can start, i.e., all states from which there are intertemporal decisions resulting in trajectories which satisfy the constraints. From the mathematical point of view, the viability kernel at initial time $t_{0}$ reads

$$
\operatorname{Viab}\left(t_{0}\right)=\left\{\begin{array}{l|l}
x_{0} \in \mathbb{X} & \begin{array}{l}
\text { there exist controls }\left(c\left(t_{0}\right), \ldots, c(T-1)\right) \\
\text { such that } \\
\forall k=1, \ldots, K, \quad \forall t=t_{0}, \ldots, T \\
\mathcal{I}_{t}^{k}(x(t), c(t)) \geq \theta_{t}^{k} \\
x(t+1)=G_{t}(x(t), c(t)) \\
x\left(t_{0}\right)=x_{0}
\end{array}
\end{array}\right\} .
$$

A basic viability problem consists in characterizing this set. In particular, the case when this kernel is empty is quite informative because it highlights the (in) consistency between the different and possibly conflicting viability constraints [Martinet and Doyen, 2007]. However, handling such a geometrical tool is not usual in economic analysis, which favors optimization approaches. However, viability kernels can be computed by means of a dynamic programming equation, that we label viable dynamic programming [De Lara and Doyen, 2008], which has close ties with the classical dynamic programming equation in dynamic optimization.

After illustrating viability kernels for the example introduced in $\S 2.1$, we will show in $\S 2.5$ how a viability problem can be expressed as a (degenerate)

dynamic optimization problem, closer to usual economic representations and efficiency.

\section{A two-species fisheries model}

In the two-species fisheries model introduced in $\S 2.1$, we consider the following goals to be satisfied:

- preservation: for all $t=t_{0}, \ldots, T$,

$$
x_{1}(t) \geq x_{1}^{b}, \quad x_{2}(t) \geq x_{2}^{b},
$$


where $x_{1}^{b} \geq 0, x_{2}^{b} \geq 0$ are minimal biomass levels, one for each species,

- production requirements: for all $t=t_{0}, \ldots, T-1$,

$$
\begin{aligned}
& c_{1}(t) x_{1}(t) \mathcal{R}_{1}\left(x_{1}(t), x_{2}(t)\right) \geq h_{1}^{b}, \\
& c_{2}(t) x_{2}(t) \mathcal{R}_{2}\left(x_{1}(t), x_{2}(t)\right) \geq h_{2}^{b},
\end{aligned}
$$

where $h_{1}^{b} \geq 0, h_{2}^{b} \geq 0$ are minimal catch levels, one for each species.

Using viable dynamic programming, we can compute explicitly the viability kernel [De Lara et al., 2012]. If the minimal biomass thresholds $x_{1}^{b}, x_{2}^{b}$ and catch thresholds $h_{1}^{b}, h_{2}^{b}$ are such that

$$
x_{1}^{b} \mathcal{R}_{1}\left(x_{1}^{b}, x_{2}^{b}\right)-x_{1}^{b} \geq h_{1}^{b} \text { and } x_{2}^{b} \mathcal{R}_{2}\left(x_{1}^{b}, x_{2}^{b}\right)-x_{2}^{b} \geq h_{2}^{b},
$$

the deterministic viability kernel is given by

$$
\operatorname{Viab}\left(t_{0}\right)=\left\{\begin{array}{l|l}
\left(x_{1}, x_{2}\right) \in \mathbb{R}_{+}^{2} & \begin{array}{l}
x_{1} \geq x_{1}^{b}, x_{2} \geq x_{2}^{b}, \\
x_{1} \mathcal{R}_{1}\left(x_{1}, x_{2}\right)-x_{1}^{b} \geq h_{1}^{b}, \\
x_{2} \mathcal{R}_{2}\left(x_{1}, x_{2}\right)-x_{2}^{b} \geq h_{2}^{b}
\end{array}
\end{array}\right\} .
$$

\subsection{Viability as a degenerate optimization problem}

Numerous formulations of viability problems in terms of optimality have been provided. In the continuous time case, Aubin [1991] especially focuses on exit time functions together with support or indicator functions for Hamiltonian characterizations. Links with the maximin criterion are pointed out in Martinet and Doyen [2007] for the Dasgupta-Heal-Solow model, or in De Lara and Doyen [2008] and Doyen and Martinet [2012] for a more general framework. We now present alternate equivalent forms of the viability problem (6) and give their interpretation. These forms will be formulated as optimization problems, although degenerate ones with no unique solution in general. This degeneracy generally makes the numerical resolution tricky. This is why our purpose is conceptual and not numerical. However, such a connection between viability and optimality also highlights the possibility to use dynamic programming to tackle the viability problem, as detailed in De Lara and Doyen [2008] and Doyen and De Lara [2010]. Such a temporal decomposition can be useful for analytical computations or numerical implementations of viability solutions, especially in the uncertain case. 


\section{Reformulating viability as an optimization problem under con- straints}

The first idea to embed the viability problem in an optimization formulation is to consider the optimization of a null (or constant) criterion under the viability constraints (5):

$$
\min _{c(\cdot) \text { satisfying (5) }} 0 \text {. }
$$

Such an optimization problem under constraints provides a viable path $(x(\cdot), c(\cdot))$ as soon as the initial state $x_{0}$ belongs to the viability kernel $\mathbb{V i a b}\left(t_{0}\right)$. In other words, the problem is not well posed outside the viability kernel, which is not satisfying. Typically, if the viability kernel is empty, such an optimization problem does not convey any information. Moreover, whenever the initial state $x_{0}$ belongs to the viability kernel $\operatorname{Viab}\left(t_{0}\right)$, several solutions and viable controls $c(\cdot)$ may exist as the zero criterion is not selective among the viable paths. This points out the degeneracy of optimization problems underlying viability issues.

\section{Reformulating viability as an optimization problem without con- straints}

Viable states belonging to the viability kernel can be characterized by solving optimization problems without constraints, using characteristic functions. ${ }^{5}$ We denote by $\mathbf{1}_{A}$ the characteristic function of the set $A$, which is equal to one when its argument belongs to $A$, and to zero otherwise:

$$
\mathbf{1}_{A}(a)=\left\{\begin{array}{lll}
1 & \text { if } & a \in A \\
0 & \text { if } & a \notin A
\end{array}\right.
$$

Using this tool, the quantity $\mathbf{1}_{\theta_{t}^{k},+\infty[}\left(\mathcal{I}_{t}^{k}(x(t), c(t))\right)$ indicates whether the constraint $k$ is satisfied at the given time or not. Using this characteristic function formulation, condition (5) can be described in multiplicative ${ }^{6}$ form, as $\prod_{k=1}^{K} \mathbf{1}_{\theta_{t}^{k},+\infty[}\left(\mathcal{I}_{t}^{k}(x(t), c(t))\right)=1$. If any of the indicator is below

\footnotetext{
${ }^{5}$ Such functions are also called indicator functions. Since we already use the term "indicator" with another meaning, we choose to speak of characteristic functions.

${ }^{6}$ An equivalent additive form is $\sum_{k=1}^{K}\left(1-\mathbf{1}_{\left[\theta_{t}^{k},+\infty[\right.}\left(\mathcal{I}_{t}^{k}(x(t), c(t))\right)\right)=0$, and the minimum form is $\min _{k=1, \ldots, K} \mathbf{1}_{\left[\theta_{t}^{k},+\infty[\right.}\left(\mathcal{I}_{t}^{k}(x(t), c(t))\right)=1$.
} 
the associated threshold, the characteristic function is equal to zero for this indicator, and the product is nil. If all the constraints are respected, the product is equal to one.

There are at least two different ways to describe the viability kernel in optimality terms using the characteristic functions. On the one hand, one can use a time-additive form, the criterion being to minimize the following value $^{7}$

$$
\sum_{t=t_{0}}^{T}\left(1-\prod_{k=1}^{K} \mathbf{1}_{\left[\theta_{t}^{k},+\infty[\right.}\left(\mathcal{I}_{t}^{k}(x(t), c(t))\right)\right),
$$

which counts the number of time periods during which (at least) one constraint is not respected along a given path. In an intergenerational perspective, it can be interpreted as the number of generations that do not achieve the objectives. It is equal to zero when all the constraints are respected at all times along the path defined by the given controls. This time-additive form has an easy interpretation based on the time of crisis criterion introduced by Doyen and Saint-Pierre [1997] and applied by Béné et al. [2001] and Martinet et al. [2007] for renewable resource management or by Martin [2004] to resilience issues. The optimal control problem associated with the criterion (11), termed minimal time of crisis, corresponds to ${ }^{8}$

$$
\mathrm{C}\left(t_{0}, x_{0}\right)=\min _{c(\cdot)} \sum_{t=t_{0}}^{T}\left(1-\prod_{k=1}^{K} \mathbf{1}_{\left[\theta_{t}^{k},+\infty[\right.}\left(\mathcal{I}_{t}^{k}(x(t), c(t))\right)\right) .
$$

It turns out that the viability kernel (6) of the problem is composed of all initial states for which the minimal time of crisis (12) is nil:

Proposition $1 x_{0} \in \mathbb{V i a b}\left(t_{0}\right) \Longleftrightarrow \mathrm{C}\left(t_{0}, x_{0}\right)=0$.

The proof follows almost by definition. Recall that our contribution is not technical but conceptual: we aim at displaying conceptual proximity between viability and optimality and we pave the way for a new criterion in the stochastic case.

On the other hand, one can use a time-multiplicative form, the criterion being to maximize the product upon time of the product of characteristic

\footnotetext{
${ }^{7}$ As we are summing nonnegative numbers, the given sum is mathematically welldefined whether the time horizon is finite, i.e., $T<+\infty$, or infinite, i.e., $T=+\infty$.

${ }^{8}$ See footnote 7 .
} 
functions $^{9}$

$$
V\left(t_{0}, x_{0}\right)=\max _{c(\cdot)} \prod_{t=t_{0}}^{T} \prod_{k=1}^{K} \mathbf{1}_{\left[\theta_{t}^{k},+\infty[\right.}\left(\mathcal{I}_{t}^{k}(x(t), c(t))\right) .
$$

As soon as one of the constraints is not respected at some time period, the criterion is equal to zero. It is equal to one when all the constraints are respected at all times, characterizing a viable state:

Proposition $2 x_{0} \in \operatorname{Viab}\left(t_{0}\right) \Longleftrightarrow V\left(t_{0}, x_{0}\right)=1$.

Even if it has little economic meaning in the deterministic case, this form will appear useful in the stochastic case that we address in the next section.

Both the criteria (12) and (13) provide the same information with respect to the characterization of the viability kernel (6). Nevertheless, the multiplicative form only gives a Boolean information, whereas the minimum time of crisis indicates, for states characterized by a strictly positive value function, the minimal number of crisis periods the economy is going to face. It thus provides a meaningful information on what happens outside the viability kernel, how to reach it and how to remain in it [Doyen and Saint-Pierre, 1997, Martinet et al., 2007, 2010]. Moreover, formulation (12), based on the concept of time of crisis, satisfies the principle of dynamic programming. ${ }^{10}$ It thus provides a useful algorithm to compute the viability kernel, and also the sets of minimal time of crisis. Martinet et al. [2010] describe such an algorithm and apply it to the issue of recovering fisheries from crisis situations.

\section{Stochastic intertemporal problem}

In this section, we proceed to the parallel description of both discounted utility and viability in a stochastic framework.

\subsection{Uncertain dynamic bio-economic model}

Consider the following discrete-time control uncertain dynamical system

$$
x(t+1)=G_{t}(x(t), c(t), w(t)), \quad t=t_{0}, \ldots, T-1, \quad x\left(t_{0}\right)=x_{0},
$$

\footnotetext{
${ }^{9}$ As we are multiplying numbers belonging to $[0,1]$, the given product is mathematically well-defined whether the time horizon is finite, i.e., $T<+\infty$, or infinite, i.e., $T=+\infty$.

${ }^{10}$ So does the time-multiplicative form (13).
} 
where $w(t) \in \mathbb{W}=\mathbb{R}^{q}$ denotes an uncertainty which affects the dynamics at time $t$. Typically, for biodiversity conservation or natural resources management issues, uncertainties can include environmental variability (weather, availability of resources or growth rate for instance). For climate change, such uncertainties might encompass uncertain emission mechanisms in the carbon cycle or natural removal of atmospheric $\mathrm{CO}_{2}$ to sinks. For the sake of simplicity, the initial state $x_{0}$ is here supposed to be deterministic and known, although, if needed, $x_{0}$ could be added to the uncertainties. We define a scenario by

$$
w(\cdot)=\left(w\left(t_{0}\right), \ldots, w(T)\right)
$$

and the set of all scenarios by

$$
\Omega=\mathbb{W}^{T-t_{0}+1} .
$$

From now on, we assume that the set $\Omega$ is equipped with a probability distribution $^{11} \mathbb{P}$ which measures the likelihood of (measurable) subsets of scenarios. ${ }^{12}$ The mathematical expectation with respect to $\mathbb{P}$ is denoted by $\mathbb{E}$.

\section{A two-species fisheries model with uncertainties}

In the two-species fisheries model presented in $\S 2.1$, we now introduce two terms $w_{1}$ and $w_{2}$ that correspond to uncertainties affecting each species biomass, respectively, through the growth factors $\mathcal{R}_{1}$ and $\mathcal{R}_{2}$. The discretetime control dynamical system we consider is:

$$
\left\{\begin{array}{l}
x_{1}(t+1)=x_{1}(t) \mathcal{R}_{1}\left(x_{1}(t), x_{2}(t), w_{1}(t)\right)\left(1-c_{1}(t)\right) \\
x_{2}(t+1)=x_{2}(t) \mathcal{R}_{2}\left(x_{1}(t), x_{2}(t), w_{2}(t)\right)\left(1-c_{2}(t)\right) .
\end{array}\right.
$$

The difference between the deterministic model (2) and the uncertain model (17) is the presence of the uncertainties variables $w_{1}$ and $w_{2}$ in the growth factors $\mathcal{R}_{1}$ and $\mathcal{R}_{2}$.

\footnotetext{
${ }^{11}$ The probability $\mathbb{P}$ is defined on the Borel product $\sigma$-field of $\mathbb{W}^{T-t_{0}+1}$. The mappings $G_{t}, \mathcal{I}_{t}^{1}, \ldots, \mathcal{I}_{t}^{k}$, and all decision rules $\mathfrak{c}_{t}$ (see below) are supposed to be measurable.

${ }^{12}$ The notation $w(\cdot)=\left(w\left(t_{0}\right), \ldots, w(T-1)\right)$ still denotes a generic point in $\Omega$; however, it may also be interpreted as a sequence of random variables when $w(\cdot)$ is identified with the identity mapping from $\Omega$ to $\Omega$.
} 
The extension of the prey-predator model of the Peruvian anchovy-hake system exposed in $\S 2.1$ is

$$
\begin{aligned}
& x_{1}(t+1)=x_{1}(t) \overbrace{(\begin{array}{c}
\left.w_{1}(t)+R-\frac{R}{\kappa} x_{1}(t)-\alpha x_{2}(t)\right) \\
x_{2}(t+1)
\end{array}=x_{2}(t) \underbrace{\mathcal{R}_{1}\left(x_{1}(t), x_{2}(t), w_{1}(t)\right)}_{\underbrace{\left(w_{2}(t)+L+\beta x_{1}(t)\right)}_{\mathcal{R}_{2}\left(x_{1}(t), x_{2}(t), w_{2}(t)\right)}\left(1-c_{2}(t)\right)} .} .
\end{aligned}
$$

\subsection{Outputs/indicators}

Main outputs of system (14) are given by indicators ${ }^{13} \mathcal{I}_{t}^{k}(x(t), c(t), w(t))$, for $k=1, \ldots, K$ and $t=t_{0}, \ldots, T$. In the uncertainty framework, an indicator $\mathcal{I}_{t}^{k}: \mathbb{X} \times \mathbb{C} \times \mathbb{W} \rightarrow \mathbb{R}$ is a state, control and uncertainty function having economic or environmental meaning.

\subsection{Decision rules}

We define a decision rule $\mathfrak{c}$ as a sequence of mappings $\mathfrak{c}_{t}: \mathbb{X} \rightarrow \mathbb{C}$, for $t=t_{0}, \ldots, T-1$. A decision rule is a (state) feedback which assigns a control $c=\mathfrak{c}_{t}(x) \in \mathbb{C}$ to any state $x$ for any time $t=t_{0}, \ldots, T-1$. With such a definition, we implicitly assume that the state is at least partially measured. ${ }^{14}$

Given a decision rule $\mathfrak{c}$, a scenario $w(\cdot) \in \Omega$, an initial state $x_{0} \in \mathbb{X}$ and an initial time $t_{0}$, the solution state path $x(\cdot)=\left(x\left(t_{0}\right), \ldots, x(T)\right)$ is well defined as the solution of the discrete-time dynamic equation

$$
\left\{\begin{aligned}
x(t+1) & =G_{t}(x(t), \mathfrak{c}(t, x(t)), w(t)) \text { with } x\left(t_{0}\right)=x_{0} \\
c(t) & =\mathfrak{c}_{t}(x(t)), \quad t=t_{0}, \ldots, T-1 .
\end{aligned}\right.
$$

The solution control path $c(\cdot)=\left(c\left(t_{0}\right), c\left(t_{0}+1\right), \ldots, c(T-1)\right)$ is the associated decision path where $c(t)=\mathfrak{c}_{t}(x(t))$.

\footnotetext{
${ }^{13}$ When the horizon $T<+\infty$, the $\mathcal{I}_{T}^{k}$ only maps the state space $\mathbb{X} \times \mathbb{W}$ towards $\mathbb{R}$ because there is no decision at time $T$. However, for the sake of homogeneity of notations, we will write $\mathcal{I}_{T}^{k}(x(T), c(T), w(T))$ for $\mathcal{I}_{T}^{k}(x(T), w(T))$.

${ }^{14}$ As a consequence, we do not consider the case where only a corrupted observation of the state is available to the decision-maker (as it may nevertheless be the case in practical situations).
} 


\subsection{Discounted expected utility}

There are plenty of criteria to make choices under risk [see, for instance, Savage, 1972], but less attention has been paid to criteria for dynamical problems mixing time and risk. The usual criterion in economics is the discounted expected utility, which is the discounted sum of present and future utilities

$$
\max _{\mathfrak{c}} \mathbb{E}\left[\sum_{t=t_{0}}^{T} \frac{1}{(1+\delta)^{t}} U\left(\mathcal{I}_{t}^{1}(x(t), c(t), w(t)), \ldots, \mathcal{I}_{t}^{K}(x(t), c(t), w(t))\right)\right],
$$

where $x(t)$ and $c(t)$ are the solution state and control paths given by (19).

Criterion (20) is built upon two well axiomatized theories, the discounted intertemporal utility [Koopmans, 1965] and the expected utility [von Neuman and Morgenstern, 1947]. This approach is widely used and offers interesting applicability properties as time consistency and dynamic programming. However, discounting makes this criterion controversial for long-run issues such as climate change (see the Stern Review [Stern, 2006] and the ongoing debate [Philibert, 2006, Weitzman, 2007, Gollier, 2008, Sterner and Persson, 2008]).

\subsection{Viable scenarios and viability probability}

We now describe how the viability approach described in $\S 2.4$ can be extended to the stochastic framework..$^{15}$

We impose viability constraints in this uncertain case as follows

$$
\mathcal{I}_{t}^{k}(x(t), c(t), w(t)) \geq \theta_{t}^{k}, k=1, \ldots, K, \quad \forall t=t_{0}, \ldots, T .
$$

For these constraints to make sense, we introduce the notion of a viable scenario. A scenario $w(\cdot)$ is said to be viable under a given decision rule $\mathfrak{c}$ if the state and control paths driven by the decision rule, introduced in §3.3, satisfy the constraints (21). In other words, if the given scenario $w(\cdot)$ materializes, the economic path defined by this decision rule is viable.

\footnotetext{
${ }^{15}$ Mathematical materials for stochastic viability can be found in Aubin and Prato [1998], Buckdahn et al. [2004] but they focus on the continuous time case. Contributions for discrete time systems are [Doyen et al., 2007, Béné and Doyen, 2008, De Lara and Doyen, 2008, De Lara and Martinet, 2009, Doyen and De Lara, 2010].
} 
For any decision rule $\mathfrak{c}$, initial state $x_{0}$, and initial time $t_{0}$, we define the set $\Omega_{\mathfrak{c}, t_{0}, x_{0}}$ of viable scenarios by:

$$
\Omega_{\mathfrak{c}, t_{0}, x_{0}}=\left\{\begin{array}{l|l}
w(\cdot) \in \Omega & \begin{array}{l}
x\left(t_{0}\right)=x_{0} \\
x(t+1)=G_{t}(x(t), c(t), w(t)) \\
c(t)=\mathfrak{c}_{t}(x(t)) \\
\mathcal{I}_{t}^{k}(x(t), c(t), w(t)) \geq \theta_{t}^{k} \\
k=1, \ldots, K \\
t=t_{0}, \ldots, T
\end{array}
\end{array}\right\} .
$$

The set $\Omega_{\mathfrak{c}, t_{0}, x_{0}}$ is the set of scenarios for which the decision rule $\mathfrak{c}$ would result in viable economic trajectories. The larger the set, the larger the number of scenarios along which the given decision rule succeeds in meeting the viability constraints. Once a probability $\mathbb{P}$ is defined on the set of scenarios, the viability problem becomes one of probability maximization, as follows.

We say that $\mathbb{P}\left(\Omega_{\mathfrak{c}, t_{0}, x_{0}}\right)$ is the viability probability associated with the initial time $t_{0}$, the initial state $x_{0}$ and the decision rule $\mathfrak{c}$. Thus, the term refers to the probability of satisfying viability constraints. Given initial time $t_{0}$ and initial state $x_{0}$, the maximal viability probability is defined by

$$
\operatorname{VP}\left(t_{0}, x_{0}\right)=\sup _{\mathfrak{c}} \mathbb{P}\left(\Omega_{\mathfrak{c}, t_{0}, x_{0}}\right)
$$

From that point of view, the stochastic viability approach aims at characterizing the decision rules that maximize the probability to comply with the constraints over time. It turns out that such maximal viability probability (23) can be characterized as the maximal expected value of the multiplicative criterion depicted in (13) for the deterministic case:

$$
\operatorname{VP}\left(t_{0}, x_{0}\right)=\max _{\mathfrak{c}} \mathbb{E}\left[\prod_{t=t_{0}}^{T} \prod_{k=1}^{K} \mathbf{1}_{\left[\theta_{t}^{k},+\infty[\right.}\left(\mathcal{I}_{t}^{k}(x(t), c(t), w(t))\right)\right] .
$$

The concept of viability kernel can then be expanded through the maximal viability probability. At a given confidence level $\beta \in[0,1]$, the stochastic viability kernel $\mathbb{V i a b}_{\beta}\left(t_{0}\right)$ is the set of initial states for which there exists a decision rule that drives the trajectories within the viability constraints (21) at least with probability $\beta$. It reads as follows [Doyen and De Lara, 2010]:

Proposition $3 x_{0} \in \mathbb{V i a b}_{\beta}\left(t_{0}\right) \Longleftrightarrow \mathrm{VP}\left(t_{0}, x_{0}\right) \geq \beta$. 
An informative case is provided by the confidence level $\beta=100 \%$ because, whenever such viability kernel $\mathbb{V i a b}_{1}\left(t_{0}\right)$ is not empty, we obtain robust viable states and decision rules in the sense that the viability constraints (21) are satisfied whatever the uncertainties occurring (up to a set of probability zero). Such worst case and pessismistic approach can also be relevant for precautionary approach as explained in Doyen and Péreau [2009].

\section{A two-species fisheries model with uncertainties}

We consider the model (17) with the following goals:

- preservation (minimal biomass levels): for all $t=t_{0}, \ldots, T$,

$$
x_{1}(t) \geq x_{1}^{b}, \quad x_{2}(t) \geq x_{2}^{b},
$$

- production requirements (minimal catch levels): for all $t=t_{0}, \ldots, T-1$,

$$
\begin{aligned}
& c_{1}(t) x_{1}(t) \mathcal{R}_{1}\left(x_{1}(t), x_{2}(t), w_{1}(t)\right) \geq h_{1}^{b}, \\
& c_{2}(t) x_{2}(t) \mathcal{R}_{2}\left(x_{1}(t), x_{2}(t), w_{2}(t)\right) \geq h_{2}^{b} .
\end{aligned}
$$

The stochastic viability kernels $\operatorname{Viab}_{\beta}\left(t_{0}\right)$ can be computed numerically by means of a dynamic programming equation associated with dynamics (17), state constraints (25) and control constraints (26) [De Lara and Doyen, 2008].

When the dynamics is given by the Lotka-Volterra model (18) with numerical values to be found in De Lara et al. [2012], and when the probability $\mathbb{P}$ on the set $\Omega$ of scenarios is such that $\left(w\left(t_{0}\right), \ldots, w(T-1)\right)$ are independent random variables (with uniform distribution on the set of observed empirical uncertainties), we obtain the stochastic viability kernels displayed in Figure 1.

\section{Practical and numerical issues}

To end, we briefly touch upon practical and numerical issues. First, the maximal viability probability and viable decision rules can theoretically be obtained by dynamic programming [De Lara and Doyen, 2008, Doyen and De Lara, 2010]. The dynamic programming equation can be solved for states of low dimension (see an example in De Lara and Doyen [2008]). Second, decision rules can be restricted to a subset and compared as was done in Doyen et al. [2007] to analyze the impact of reserve size in fishery management. Third, under specific properties such as monotonicity of dynamics and 


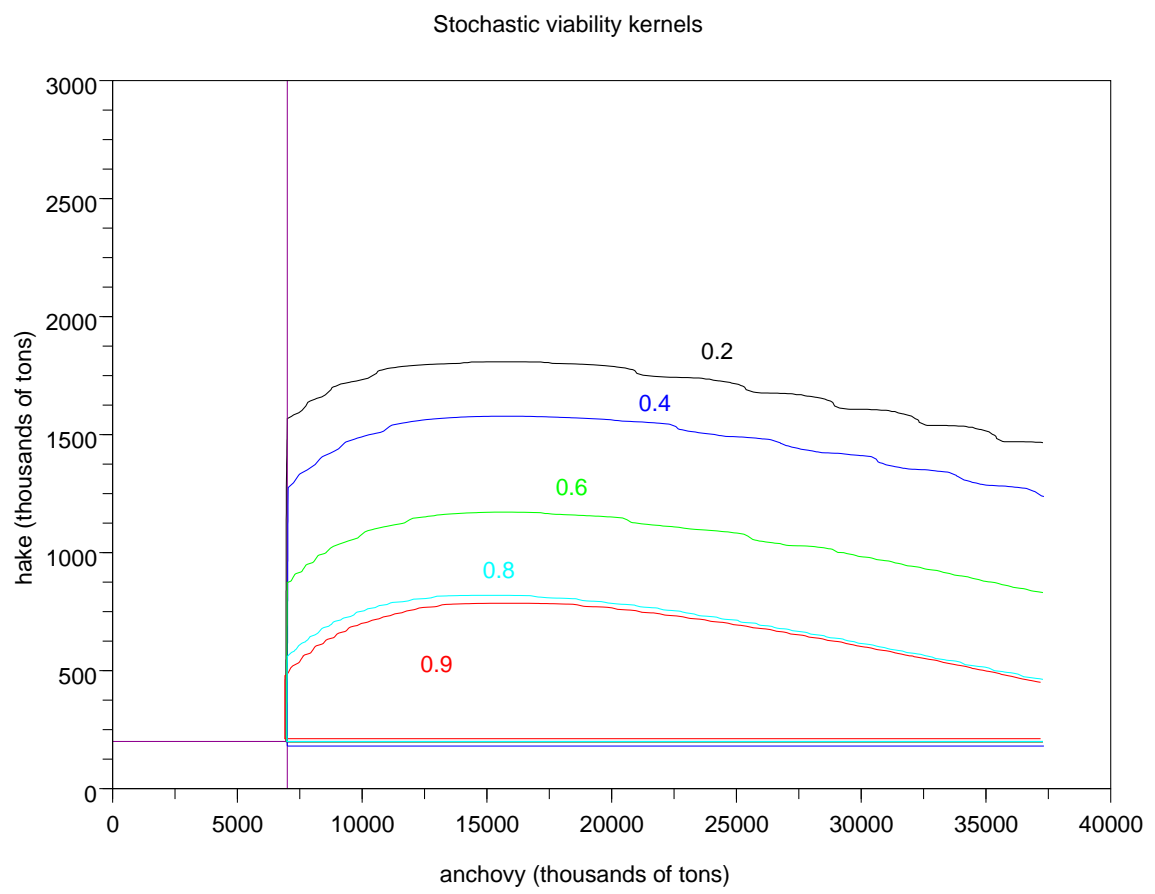

Figure 1: Stochastic viability kernels $\operatorname{Viab}_{\beta}\left(t_{0}\right)(\beta=0.2,0.4,0.6,0.8,0.9)$ for the hake-anchovy fisheries model (17) with the viability constraints (25) and (26)

outputs with respect to state and control variables, a maximal viable decision rule can be characterized and explicitly described, as in De Lara and Martinet [2009], where a fisheries example with a state of dimension 18 is treated.

\section{Discussion and conclusion}

When dealing with environmental issues, such as biodiversity management or climate change, both risk and intertemporal issues are to be considered. The main economic criterion combining risk and time is the discounted expected utility. The controversy on the choice of the discount rate stands as an important point in the debate on the economics of sustainability. However, 
even with a low discount rate, discounting is criticized as extinction of a species can be optimal [Clark, 1990] or as long-run damages and benefits are neglected due to exponential discounting. ${ }^{16}$

In practice, the climate change issue is tackled using quantities as emissions targets, upper limit for long-run GHG concentration or mean temperature increment, namely using indicators and thresholds in our terminology. Such an approach is also widespread in biodiversity conservation or natural resources management, with minimal preservation thresholds for abundances. As recalled in Sect. 1, the International Council for the Exploration of the Sea (ICES) precautionary approach [ICES, 2004] aims at conserving fish stocks and fisheries on the grounds of spawning stock biomass and fishing mortality.

The present paper points out how viability, and especially stochastic viability, can contribute to scientific environmental debates. Viability aims at defining the conditions for sustainability constraints to be satisfied over time. Using the stochastic viability approach to address sustainability issues is interpreted as maximizing the probability to respect constraints, and to avoid exceeding potentially dangerous thresholds.

Stochastic viability focuses on the decision rules that minimize the risk to violate the constraints, and thus aims at avoiding related catastrophic outcomes. Consider, for example, ecosystem management as inspired by Population Viability Analysis [Morris and Doak, 2003] which minimizes extinction risks. In that case, stochastic viability would favor decision rules such that constraints related to both biodiversity and ecosystem services are satisfied with high probability. In other words, stochastic viability may be seen as a method to control the tails of a damages distribution. Comparing maximal viability probabilities for different potentially catastrophic issues (climate change, nuclear energy, etc.) is one possible answer to the programmatic conclusion by Weitzman [2007] on the importance of comparing tails of distributions.

Acknowldgements. The authors are indebted to the Editor and to two Reviewers for their useful comments.

\footnotetext{
${ }^{16}$ An exponential increase in relative prices may nevertheless compensate the exponential decrease [Boiteux, 1976, Philibert, 1999, 2006, Sterner and Persson, 2008]
} 


\section{References}

M. Allais. Le comportement de l'homme rationnel devant le risque: Critique des postulats et axiomes de l'école américaine. Econometrica, 21(4):503546 , october 1953 .

J-P. Aubin. Viability Theory. Birkhäuser, Boston, 1991.

J.-P Aubin and G. Da Prato. The viability theorem for stochastic differential inclusions. Stochastic Analysis and Applications, 16:1-15, 1998.

Stefan Baumgärtner and Martin F. Quaas. Ecological-economic viability as a criterion of strong sustainability under uncertainty. Ecological Economics, 68(7):2008 - 2020, 2009.

C. Béné and L. Doyen. Contribution values of biodiversity to ecosystem performances: A viability perspective. Ecological Economics, 68(1-2):14$23,2008$.

C. Béné, L. Doyen, and D. Gabay. A viability analysis for a bio-economic model. Ecological Economics, 36:385-396, 2001.

M. Boiteux. À propos de la "critique de la théorie de l'actualisation telle qu'employée en France". Revue d'Économie Politique, 5, 1976.

R. Buckdahn, M. Quincampoix, C. Rainer, and A. Rascanu. Stochastic control with exit time and contraints, application to small time attainability of sets. Applied Mathematics and Optimization, 49:99-112, 2004.

U. Chakravorty, M. Moreaux, and M. Tidball. Ordering the extraction of polluting nonrenewable resources. American Economic Review, 98(3):11281144, 2008.

G. Chichilnisky. An axiomatic approach to sustainable development. Social Choice and Welfare, 13(2):219-248, 1996.

A. Cissé, S. Gourguet, L. Doyen, F. Blanchard, and J.-C. Péreau. A bioeconomic model for the ecosystem-based management of the coastal fishery in French Guiana. Environment and Development Economics, 18:245-269, June 2013. 
C. W. Clark. Mathematical Bioeconomics. Wiley, New York, second edition, 1990.

P. Dasgupta and G. Heal. The optimal depletion of exhaustible resources. Review of Economic Studies, 41:1-28, 1974. Symposium on the Economics of Exhaustible Resources.

M. De Lara and V. Martinet. Multi-criteria dynamic decision under uncertainty: A stochastic viability analysis and an application to sustainable fishery management. Mathematical Biosciences, 217(2):118-124, February 2009 .

Michel De Lara and Luc Doyen. Sustainable Management of Natural Resources. Mathematical Models and Methods. Springer-Verlag, Berlin, 2008.

Michel De Lara, Eladio Ocaña Anaya, Ricardo Oliveros-Ramos, and Jorge Tam. Ecosystem viable yields. Environmental Modeling \& Assessment, 17 (6):565-575, 2012.

L. Doyen and M. De Lara. Stochastic viability and dynamic programming. Systems and Control Letters, 59(10):629-634, October 2010.

L. Doyen and V. Martinet. Maximin, viability and sustainability. Journal of Economic Dynamics and Control, 36(9):1414-1430, 2012.

L. Doyen and P. Saint-Pierre. Scale of viability and minimum time of crisis. Set-valued Analysis, 5:227-246, 1997.

L. Doyen, M. De Lara, J. Ferraris, and D. Pelletier. Sustainability of exploited marine ecosystems through protected areas: a viability model and a coral reef case study. Ecological Modelling, 208(2-4):353-366, November 2007.

L. Doyen, O. Thébaud, C. Béné, V. Martinet, S. Gourguet, M. Bertignac, S. Fifas, and F. Blanchard. A stochastic viability approach to ecosystembased fisheries management. Ecological Economics, 75(0):32 - 42, 2012.

Luc Doyen and Jean-Christophe Péreau. The precautionary principle as a robust cost-effectiveness problem. Environmental Modeling and Assessment, 14(1):127-133, 2009.

L.E. Dubbins and L.J. Savage. How to gamble if you must. Inequalities for stochastic processes. McGraw-Hill Education, 1965. 
D. Ellsberg. Risk, ambiguity, and the Savage axioms. Quartely Journal of Economics, 75:643-669, 1961.

Christian Gollier. Discounting with fat-tailed economic growth. Journal of Risk and Uncertainty, 37(2):171-186, December 2008.

S. Gourguet, C. Macher, L. Doyen, O. Thébaud, M. Bertignac, and O. Guyader. Managing mixed fisheries for bio-economic viability. Fisheries Research, 140:46-62, 2013.

Pierre-Yves Hardy, Christophe Béné, Luc Doyen, and Anne-Marie Schwarz. Food security versus environment conservation: A case study of Solomon Islands' small-scale fisheries. Environmental Development, 8:38-56, October 2013.

G. Heal. Valuing the Future, Economic Theory and Sustainability. Columbia University Press, New York, 1998.

R. Howarth. Sustainability under uncertainty: A deontological approach. Land Economics, 71(4):417-427, 1995.

ICES. Report of the ICES advisory committee on fishery management and advisory committee on ecosystems, 2004. ICES Advice, 1, ICES, 2004. $1544 \mathrm{pp}$.

Daniel Kahneman and Amos Tversky. Prospect theory: An analysis of decision under risk. Econometrica, 47(2):263-292, 1979.

T. Koopmans. On the concept of optimal economic growth. Academia Scientiarium Scripta Varia, 28:225-300, 1965.

J. Krawczyk and K. Kim. Satisficing solutions to a monetary policy problem: a viability theory approach. Macroeconomic Dynamics, 13(1), 2009.

Lola L. Lopes. When time is of the essence: Averaging, aspiration, and the short run. Organizational Behavior and Human Decision Processes, 65(3): 179-189, March 1996.

K.G. Mäler. Environment, uncertainty, and option values. Beijer Institute Working Paper, 2002. 
S. Martin. The cost of restoration as a way of defining resilience: a viability approach applied to a model of lake eutrophication. Ecology and Society, $9(2), 2004$.

V. Martinet and L. Doyen. Sustainable management of an exhaustible resource: a viable control approach. Resource and Energy Economics, 29(1): 19-37, 2007.

V. Martinet, L. Doyen, and O. Thébaud. Defining viable recovery paths toward sustainable fisheries. Ecological Economics, 64(2):411-422, 2007.

Vincent Martinet, Olivier Thébaud, and Alain Rapaport. Hare or Tortoise? Trade-offs in recovering sustainable bioeconomic systems. Environmental Modeling and Assessment, 15(6):503-517, 2010.

W. F. Morris and D. F. Doak. Quantitative Conservation Biology: Theory and Practice of Population Viability Analysis. Sinauer Associates, 2003.

L. Mouysset, L. Doyen, and F. Jiguet. From population viability analysis to coviability of farmland biodiversity and agriculture. Conservation Biology, 28(1):187-201, 2014.

J.-C. Péreau, L. Doyen, L.R. Little, and O. Thébaud. The triple bottom line: Meeting ecological, economic and social goals with individual transferable quotas. Journal of Environmental Economics and Management, 63(3):419 $-434,2012$.

Cédric Philibert. The economics of climate change and the theory of discounting. Energy Policy, 27(15):913-927, December 1999.

Cédric Philibert. Discounting the future. In D. Pannell and S. Schilizzi, editors, Discounting and Discount Rates in Theory and Practice, chapter 10, pages 136-148. Edward Elgar, Cheltenham, 2006.

L. J. Savage. The Foundations of Statistics. Dover, New York, second revised edition, 1972.

H. Simon. A behavioral model of rational choice. In Models of Man, Social and Rational: Mathematical Essays on Rational Human Behavior in a Social Setting. Wiley, New York, 1957. 
R. M. Solow. Intergenerational equity and exhaustible resources. Review of Economic Studies, 41:29-45, 1974. Symposium on the Economics of Exhaustible Resources.

Nicholas Stern. The Economics of Climate Change. Cambridge University Press, 2006.

Thomas Sterner and U. Martin Persson. An Even Sterner Review: Introducing Relative Prices into the Discounting Debate. Rev Environ Econ Policy, 2(1):61-76, 2008.

Amos Tversky and Daniel Kahneman. Advances in prospect theory: Cumulative representation of uncertainty. Journal of Risk and Uncertainty, 5 (4):297-323, October 1992.

J. von Neuman and O. Morgenstern. Theory of games and economic behaviour. Princeton University Press, Princeton, second edition, 1947.

Martin L. Weitzman. A review of the Stern review on the economics of climate change. Journal of Economic Literature, 45(3):703-724, September 2007. 